Revue des arts et médiations humaines

\title{
Des petites madeleines et des tweets
}

Le Madeleine Project de Clara Beaudoux

\section{Anaïs Guilet}

\section{(2) OpenEdition}

Journals

Édition électronique

URL : https://journals.openedition.org/hybrid/356

DOI : 10.4000/hybrid.356

ISSN : 2276-3538

Traduction(s) :

Madeleines \& tweets - URL : https://journals.openedition.org/hybrid/439 [en]

Éditeur

Presses universitaires de Vincennes

Référence électronique

Anaiis Guilet, «Des petites madeleines et des tweets », Hybrid [En ligne], 5 | 2018, mis en ligne le 18 décembre 2018, consulté le 13 avril 2022. URL : http://journals.openedition.org/hybrid/356 ; DOI : https://doi.org/10.4000/hybrid.356

Ce document a été généré automatiquement le 13 avril 2022.

Revue Hybrid 


\title{
Des petites madeleines et des tweets
}

\author{
Le Madeleine Project de Clara Beaudoux
}

\section{Anaïs Guilet}

Clara Beaudoux est journaliste à Radio France et réalisatrice de plusieurs webdocumentaires. Elle vit à Paris et trouve dans la cave de son nouvel appartement les affaires de Madeleine, l'ancienne locataire des lieux. Pendant une semaine, en novembre 2015, sur Twitter ${ }^{2}$, elle mènera l'enquête avec tendresse et pudeur, et mettra à jour la vie de la presque centenaire: quelques mots, des images, le portrait de Madeleine en même temps que d'une époque révolue, se dessinent. Qualifié tour à tour de «feuilleton 2.0 » ou de " tweet documentaire " ${ }^{3}$, le Madeleine Project compose un récit de vie, tweet après tweet, comme une boîte ou lettre ouvertes. Le lecteur peut découvrir la personnalité et l'époque de ce personnage inédit qu'est Madeleine de deux manières : soit au fil de la publication des tweets pendant chacune des quatre saisons orchestrées avec de plus en plus d'acuité par Clara Beaudoux, ces quatre saisons constituant autant de performances d'écriture narrative transmédias. Soit le lecteur découvre le Madeleine Project hors de ces temps de performance à travers plusieurs médias « agrégateurs », principalement le site web du projet ${ }^{4}$, Storify ${ }^{5}$, un premier livre regroupant les deux premières saisons, paru aux Éditions du sous-sol en 2016, puis un livre de poche sorti en 2017 compilant l'intégralité des quatre saisons. Les objets et papiers que Madeleine a archivés dans des dizaines de boîtes méticuleusement étiquetées sont les points de départ de l'entreprise narrative de Beaudoux. En cela, le contenu de la cave s'accorde avec ce que dit Herman Parret des archives, qui pour lui

[...] livrent le non-dit, qu'elles forcent une brèche dans le tissu des jours quotidiens et sont tendues vers l'inattendu. Est-ce un sentiment naïf que par le décodage de l'archive on déchire un voile, on traverse l'opacité d'un savoir et on accède à l'essentiel des êtres et des choses ${ }^{6}$ ?

2 «[...] [L]'archivage, comme une mise à nu ${ }^{7} »$, compte tenu de tout le questionnement éthique qu'il aura posé à Beaudoux, permet d'interroger la mémoire mais surtout la trace, qui se fait ici poétique. Dans Temps et récit 3, Paul Ricœur évoquant la symbolique de l'absent que figurent nos ancêtres et nos descendants, voit dans la trace un mixte et même un connecteur entre le temps physique et le temps vécu que la phénoménologie heideggérienne avait dissociés. Marque laissée par quelque chose, par quelqu'un, indice 
perceptible d'une présence passée, émanation du référent, la trace procède de ce « ça a été " souligné par Roland Barthes dans LaChambre claire ${ }^{8}$. La trace est ce que présuppose tout document et donc toute archive, elle est tracée ${ }^{9}$ dans le cadre du Madeleine Project, un parcours qui déclenche l'anamnèse de Madeleine et donc, les deux étant intrinsèquement reliés, provoque le récit. Cette articulation entre mémoire et récit dans le Madeleine Project est bivalente: il y a la mémoire de Madeleine que reconstruit Beaudoux à travers la mise en récit de la découverte de ses archives sur les réseaux sociaux; mais il y a également la mémoire même de la performance qu'est le Madeleine Project. Cette dualité permet d'interroger la trace à la fois dans sa dimension physique et numérique, toutes deux faisant l'objet d'une mise en récit, en tant que représentation d'un événement, et d'une remédiatisation. Car, en effet, comme le note Sybille Kramer :

Les traces n'apparaissent que dans la mesure où une forme existante est effacée puis reconfigurée sous l'effet d'une réécriture. [...] La sémantique de la trace ne se déploie qu'au sein d'une " logique » de la narration, dans laquelle la trace se dote de son propre « lieu narré » [...]. Les traces représentent en somme le lieu où les choses muettes «se mettent à parler » grâce à notre intuition ${ }^{10}$.

\section{Performance et trace}

\section{Le projet éditorial de Beaudoux}

Jean Baudrillard ${ }^{11}$ et Paul Virilio ${ }^{12}$ ont démontré comment la télévision depuis la première guerre du Golfe - mais on pourrait y ajouter Internet - ont bouleversé notre rapport au monde du fait de leur capacité à reporter tout événement dans une quasi simultanéité avec son déroulement. À rebours de cette "compression temporelle ${ }^{13}$ ", Beaudoux reporter s'intéresse aux événements d'une vie passée. Mais elle le fait sous la forme d'une performance d'écriture qui se déploie principalement sur la plateforme de microblogging Twitter, à coup de 140 caractères, de photographies, d'hyperliens vers des chansons ou des vidéos: créant dans un même mouvement un happening, un événement journalistico-littéraire sous forme de feuilleton transmédia. Le Madeleine Project sur Twitter fait performance. Sans aborder les problèmes de définitions inhérents à la variété de pratiques que recoupe la performance, ou la pluralité de disciplines qui se sont appropriées le terme, nous proposons ici de désigner par " performance » tout événement artistique produisant des gestes, des actes, ayant lieu le plus souvent en public, et dont le déroulement temporel constitue l'œuvre même. La performance peut être plus ou moins improvisée, mais chaque occurrence reste unique. La présence du performeur (qui peut être physique ou médiatisée) y est généralement centrale. Passant outre l'équivocité essentielle du terme de performance et renvoyant aux essais fondateurs de Roselee Goldberg, Cynthia Carr ou Arnaud Label-Rojoux ${ }^{14}$, il s'agira de nous concentrer sur la manière dont Beaudoux la pratique en acte, ou plus précisément en tweets.

4 Même si Beaudoux déclare à plusieurs reprises dans les journaux que rien n'était prémédité et que sa démarche était à l'origine tout à fait indépendante de son travail de journaliste pas plus qu'elle ne s'inscrivait dans une démarche littéraire, il n'empêche que le Madeleine Project dans ses prémices est désir de récit: le tweet inaugural sur le compte de Clara Beaudoux le démontre avec force, il s'agit de raconter une histoire: «Voilà plus de deux ans que je veux raconter cette histoire. Alors je vais tenter de le 
faire ici cette semaine ${ }^{15}$. $"$ Cette histoire s'élabore comme un work in progress, pour le lecteur comme pour Beaudoux. C'est dans le courant de la première semaine de publication des tweets, que s'élabore le projet éditorial de Beaudoux. Dans la matinée du premier jour (11 h 30$)$ le hashtag \#madeleineProject apparaît, pour devenir quasi

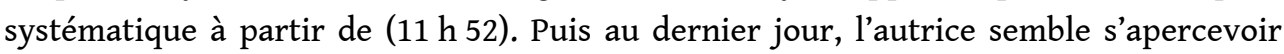
que le travail d'investigation doit se poursuivre et promet une seconde salve de tweets. Elle se met alors à parler de saison 1 , sur le modèle des populaires séries télévisées, pour décrire cet enchaînement de publications consacrées aux archives personnelles de Madeleine, qui s'étale entre le 2 et le 6 novembre 2015. Pour la saison 2, qui débute le 8 février 2016, Beaudoux sortira de la cave pour partir à la rencontre de ceux qui ont connu Madeleine: son filleul, un couple d'anciens voisins, un commerçant. Puis la

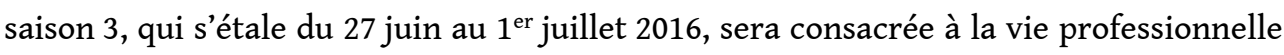
de Madeleine, à la visite de Beaudoux à Bourges, ville natale de la vieille dame, ainsi qu'à approfondir les informations sur Loulou, son fiancé mort à la guerre. La saison 4, quant à elle, s'emploiera à décrire la vie d'institutrice de Madeleine et à essayer de retrouver quelques-uns de ses anciens élèves.

\section{Un récit communautaire}

5 Pendant chacune de ces saisons, Beaudoux publie parfois plus d'une vingtaine de tweets par jour. La première saison en compte 265. Elle les envoie d'affilée, en général autour de midi, à quelques secondes d'intervalle les uns des autres. En tant que performance, les saisons ont une durée limitée dans le temps, et parce qu'elles se déploient sur les réseaux, elles sont publiques, permettant d'intégrer les réactions et commentaires des internautes, de jauger leur intérêt et donc de s'y adapter. La nécessité du hashtag pour favoriser une lecture des messages du Madeleine Project, qui autrement seraient noyés dans le flux des utilisateurs, témoigne de ce souci de partage et de cet apprentissage de la gestion de l'outil par Beaudoux. Cet hashtag a l'avantage de pouvoir également être utilisé par les lecteurs du Madeleine Project qui n'hésitent pas à «liker ", retweeter et répondre à ses publications.

6 Au-delà du «buzz» qu'il aura produit, l'aspect communautaire du projet, son édification collective sont fondamentaux dans la mesure où ils construisent l'universalité et la poéticité même du récit de Madeleine, entre documentaire, témoignage et fiction. Les saisons 3 et 4 avec l'intervention des élèves de CM2 de l'école Jean Macé d'Aubervilliers, où Madeleine a été institutrice, pour retrouver certains de ses anciens élèves l'exemplifient très bien: le Madeleine Project est bâti comme une enquête collective, une sorte de Serial des réseaux sociaux. Serial est une émission de radio américaine "podcastée » à partir d'octobre 2014 dans le monde entier. Il s'agit d'une enquête sur un meurtre commis en 1999, qui est racontée comme une série télévisée. Les auditeurs de Serial, pris de passion, se sont mêlés à l'enquête, comme ceux du Madeleine Project après lui. Les internautes viennent ainsi à l'aide pour identifier les objets incongrus découverts dans la cave, envoyer des photos des lieux évoqués par Madeleine dans ses lettres, ou pour demander et réaliser une de ses recettes de cuisine.

\section{Archiver la performance}

7 Pour ceux qui n'auraient pu suivre les tweets en synchronie avec leur publication, des traces de la performance sont proposées sous deux formes principales d'agrégation: 
Storify sur le Web et deux livres. Le site web quant à lui ne fait que rassembler les liens Storify vers chaque saison. Storify est une application qui permet, comme son nom le laisse entendre, de raconter une histoire. Il rassemble, dans une timeline multimédia, les publications issues d'un réseau social ou autres sources tout en rétablissant leur ordre chronologique, le flux usuel de Twitter étant antéchronologique. La narration peut de ce fait inclure des hyperliens vers des pages web, des images puisées sur Instagram ou Flickr, des vidéos de YouTube, ainsi que des contenus provenant d'autres réseaux sociaux ou sites. Storify est donc un outil de curation des contenus web et un outil de storytelling ${ }^{16}$.

8 Le livre publié aux Éditions du sous-sol en mai 2016, pour ne s'en tenir qu'à lui, peut être considéré comme une version imprimée homothétique du Storify des saisons 1 et 2 $\mathrm{du}$ Madeleine Project. Dans un processus de remédiatisation à rebours de la numérisation : l'impression du Web. Le livre est d'ailleurs présenté au verso de la page de grand titre dans son achevé d'imprimé comme "une adaptation [du] hashtag \#MadeleineProject ». Le livre propose deux à quatre tweets par page et reproduit les images twittées le cas échéant. Les tweets, qui sont regroupés non en chapitres mais par jour de la performance, sa temporalité étant ainsi mise en valeur, sont reproduits en contexte, comme si une capture d'écran avait été effectuée, et ils sont entourés du blanc de la page. Dans les faits, ces captures sont retravaillées, pour faire apparaître le logo Twitter qui détient les droits des publications - expliquant ainsi le copyright du colophon, et pour faire disparaître les avatars des internautes qui ont partagé le message. Ne reste ainsi que l'avatar de Beaudoux, l'heure et la date précise de la publication, ainsi que le compteur de « likes » et retweets au moment de la publication.

Le livre construit donc un " effet » Twitter ${ }^{17}$, qui ne se joue pourtant qu'en surface. Les liens, les vidéos, les morceaux soundcloud n'y subsistent qu'en tant que pures figures du passé numérique du récit puisqu'ils ne sont plus cliquables : ils ne sont qu'une trace, la manifestation de l'absence du média numérique originel. Ainsi, à la page 42 du livre, on trouve un lien et une vidéo vers la chanson "Garde ton cœur Madeleine ", citée dans un des carnets où la vieille dame recopiait des paroles. Ces effets sont une manière, pour Beaudoux et l'éditeur, de "garder la spécificité du média, de ces tweets, de ce reportage-photo où le texte avance en légende, plutôt que d'obliger le nouveau format à s'adapter à l'ancien, absurde décalage et anachronisme dénaturant le projet initial ${ }^{18}$ ". Ces effets soulignent le statut de remédiatisation du Madeleine Project, et donc son travail d'archéologie narrativo-médiatique - l'archéologie s'étant elle-même définie comme " un paradigme de l'indice à l'œuvre ${ }^{19} »$. Il s'agit en effet pour le livre de faire trace du récit élaboré sur Storify, qui lui-même reconstituait le récit effectué sur Twitter, qui lui-même composait le portrait de Madeleine tiré de ses archives. Toute lecture du Madeleine Project impose ce va-et-vient médiatique, c'est en cela que l'œuvre est transmédia. Elle construit donc sa poétique de la trace sur un travail de décontextualistaion/recontextualisation à plusieurs niveaux (thématique, narratif et médiatique) et, comme le note Bruno Bachimont :

Il est bien clair que la décontextualisation d'un contenu est inhérente à l'acte même de son inscription. À l'immanence de la communication ici et maintenant, dans son contexte natif, l'inscription donnant naissance à un contenu fixé sur support est d'emblée la décontextualisation de ce contenu vis-à-vis de l'interaction ou de l'évènement initial dont il est l'empreinte, l'enregistrement, la consignation, l'inscription. Les minutes d'un procès ne sont pas ce dernier; un texte relatant un discours ne permet pas de le revivre tel qu'il a été prononcé, etc. Cette incapacité n'est pas un défaut, mais la qualité recherchée, car par cette décontextualisation, 
on permet une recontextualisation future, dans un contexte de lecture ou de consultation à venir, différent du contexte de production. Les inscriptions sont de manière générale conçues pour cela, pour permettre de remobiliser une trace à nouveau, et donc à nouveau fraîche, interprétative bien sûr ${ }^{20}$.

10 C'est tout l'enjeu du Madeleine Project: rafraîchir la trace de Madeleine, et des différentes étapes médiatiques du travail de Beaudoux dans un élan constant contre l'oubli. L'autrice ne s'y trompe pas, d'ailleurs, qui expliquant les raisons d'être de son livre, les mêle à l'impératif de la mise en récit :

S'agissait-il de se lancer dans une bataille contre l'oubli ? Pourquoi désormais imprimer tous ces tweets, coucher sur papier l'immatériel ? Pour garder la mémoire de ta mémoire Madeleine? Pour garder une trace? Mais que restera-t-il de nous deux $^{21}$ ?

\section{Mémoire et récit}

\section{D'une cave comme d'une tasse de thé}

Madeleine, n'aurait alors pu s'appeler autrement, elle qui est l'objet sinon d'une véritable réminiscence - puisque Beaudoux ne peut en posséder de souvenir, du moins d'un travail de mémoire à partir duquel se déploie toute l'ampleur du récit. En cela, le Madeleine Project est tout à fait proustien. Et si le rapprochement semble facile, il n'en reste pas moins juste quand

[...] d'un passé ancien rien ne subsiste, après la mort des êtres, après la destruction des choses, seules, plus frêles mais plus vivaces, plus immatérielles, plus persistantes, plus fidèles, l'odeur et la saveur restent encore longtemps, comme des âmes, à se rappeler, à attendre, à espérer, sur la ruine de tout le reste, à porter sans fléchir, sur leur gouttelette presque impalpable, l'édifice immense du souvenir ${ }^{22}$.

Chacun de ces gestes effectués dans la cave par Beaudoux, de ces valises ouvertes, de ces photos postées, de ces tweets, sont aussi triviaux que transcendants en ce qu'ils font apparaître toute une personnalité, une époque, de la même manière que Combray est sorti "ville et jardins", de la tasse de thé du narrateur de la Recherche. Récits journalistique, historique ou de fiction, tous reposent sur une même logique de configuration du temps grâce à l'acte de narration ${ }^{23}$. Le travail de Beaudoux renoue avec cette dimension quasi anthropologique du récit désigné comme une catégorie de la saisie humaine du monde. Ceci ne l'empêche cependant pas d'obéir également aux définitions qu'en ont proposées la linguistique textuelle ou la narratologie: tous les critères strictement structurels s'y retrouvent : clôture, organisation avec début, milieu et fin, unité d'action et d'intention, principe de causalité et de nécessité narrative, conclusion congruente, etc. ${ }^{24}$.

\section{Une intrigue mythologisante}

Le Madeleine Project est ainsi élaboré comme une véritable enquête, dont l'intrigue est faite de rebondissements et de découvertes dramatiques. Le récit a été par ailleurs préalablement organisé par Beaudoux, qui a trié attentivement ce qu'elle souhaitait ou non divulguer et ménage ainsi sa progression. $\mathrm{Si}$, comme beaucoup des œuvres de Sophie Calle ${ }^{25}$ du début des années 1980 - que Beaudoux cite d'ailleurs souvent en entretien -, Le Madeleine Project s'inscrit dans une poétique de la trace, Beaudoux se veut plus pudique que l'artiste. Elle préserve l'anonymat de Madeleine et de son 
entourage et sélectionne, pour ne pas dire censure, beaucoup de ses trouvailles afin de préserver leur intimité. Dans le cadre de cette attention à la mise en récit, elle travaille dans la première saison à préciser d'abord l'identité de Madeleine, elle pose le personnage : ce à quoi elle ressemble, après quoi elle donne des informations sur sa famille, ses parents. La saison 1 culmine avec la découverte d'une valise dans laquelle se trouvent tous les documents en rapport avec Loulou, principalement des lettres et des photographies, dont on découvre au fil des tweets qu'il était le fiancé de Madeleine et que ce dernier est mort pendant la guerre. Il avait 31 ans. L'histoire de Loulou et Madeleine, à l'instar de l'ensemble du Madeleine Project sont, comme le relève la préface du livre, la «preuve mouvante de l'inventivité du réel et du romanesque inhérent à toute vie, y compris la plus anonyme ${ }^{26} »$. Cette relation romantique avec Loulou, dans sa dimension dramatique, ménage par ailleurs la tension narrative, non seulement au sein de l'intrigue de la saison 1, mais également entre les saisons 1 et 2. Cette tension narrative établit résolument la succession de ses tweets comme une intrigue. Pour Baroni, elle est le

[...] phénomène qui survient lorsque l'interprète d'un récit est encouragé à attendre un dénouement, cette attente étant caractérisée par une anticipation teintée d'incertitude qui confère des traits passionnels à l'acte de réception. La tension narrative sera ainsi considérée comme un effet poétique qui structure le récit et l'on reconnaîtra en elle l'aspect dynamique ou la «force » de ce que l'on a coutume d'appeler une intrigue ${ }^{27}$.

14 La saison 1 se clôt sur la dernière lettre de Madeleine à Loulou, jamais arrivée à destination. Elle est datée du 6 juin 1940. Madeleine y évoque leur première fois que Beaudoux passe sous silence. Par pudeur certes, mais aussi pour ménager suffisamment d'interstices dans lesquelles l'imagination du lecteur peut s'engouffrer, où le processus d'identification peut se déployer. Les commentaires prouvent l'efficacité de cette stratégie puisque tel internaute témoignera que sa propre grand-mère s'appelle Madeleine, tel autre qu'il avait une voisine qui lui ressemblait beaucoup. L'amour, la mort, la guerre, victimes et héros: tous les ingrédients d'une scénarisation mythologisante sont rassemblés, qui permettent à l'histoire de toucher à l'universel en même temps qu'elle renvoie chaque lecteur à ses propres caves et greniers. Voilà qui nous ramène à l'importance du muthos ou mise en intrigue qui depuis Aristote est mimesis d'une action, non pas simple imitation mais représentation, agencement de faits en système, imitation active, créatrice d'actions humaines et, par-là, d'expériences personnelles indissociables de la temporalité.

\section{Sous l'égide de Mnémosyne}

Loulou meurt à 31 ans, l'âge de Beaudoux au moment de cette découverte. C'est elle qui le dit $^{28}$. Ainsi, en même temps que le portrait de Madeleine se dessine, c'est la présence de la narratrice qui s'affirme. Le Madeleine Project double alors l'écriture biographique d'une autobiographie en filigrane, démontrant, encore une fois de manière très proustienne, le rôle de la narration dans la quête et la constitution de l'identité. Clara Beaudoux, de plus en plus impliquée au fil des tweets, se prend à révéler des informations la concernant, souvent entre parenthèses, dans une sorte d'aparté: comme Madeleine, elle aime aller à la patinoire ${ }^{29}$ et affectionne les petits carnets de note ${ }^{30}$. Cette proximité, pour ne pas dire intimité, entre les deux femmes se construit aussi dans le dialogue que Beaudoux engage avec Madeleine. À partir du second jour de 
la première saison ${ }^{31}$, apparaissent des tweets rédigés à la deuxième personne du singulier, en même temps que la première personne du singulier se fait de plus en présente. Beaudoux le déclare, "grâce au Madeleine Project je me suis mise à dire je ${ }^{32}$ ". Elle se glisse littéralement dans la vie de Madeleine, qui semble lui aller comme un gant. Dans une de ses publications, elle enfile d'ailleurs littéralement les gants de Madeleine et s'adressant directement à la vieille dame, prend sa main en photo et l'accompagne du tweet suivant: «Un drôle de cap d'engagement physique dans le projet, Madeleine : $)^{33} »$.

16 Le travail d'écriture de Beaudoux renoue ainsi avec Mnemosynè. Mnemosynè est Mémoire, la déesse mère des neuf muses, qui préside à la haute poésie. Poésie et mémoire sont si étroitement liées que pour Homère, versifier c'était se souvenir :

La mémoire est placée au commencement, elle est la matrice où s'inventent tous les arts humains, où naissent toutes les fabriques de l'homme, y compris la fabrication des idées. Mémoire et invention sont assimilées dans ce qui serait appelé aujourd'hui la « créativité $»^{34}$.

Chez Aristote, la remémoration est investigatio, véritable travail de pistage, d'actualisation des «marques» de la mémoire, impliquant nécessairement une interprétation qui dans son sillage entraîne "toute la rhétorique de l'invention et mobilise la faculté créatrice de l'imagination ${ }^{35} »$.

18 Ainsi, le récit proposé par Beaudoux repose sur un exercice de mémoire, de lecture d'archive, d'enquête et de mise en récit, mise en média: un véritable travail de création. Madeleine y devient personnage, qui de par ses contours anonymés, comme de par le récit mythologisant de Beaudoux, a tout du témoin, ici historique, qu'affectionne le journalisme narratif. Elle est à la fois ancrée dans le réel et marqueur pour l'imaginaire. Le Madeleine Project, dans sa version éditée, est identifié comme « un reportage de Clara Beaudoux », et sous-titré sur sa page de titre: "Feuilleton/NonFiction $^{36}$ ». Il est publié aux Éditions du sous-sol dédiées à l'écriture documentaire et à la non-fiction. Il s'agit d'un récit hybride, aux frontières du journalisme et de la littérature. En tant que tel, il s'inscrit pleinement dans cette vogue du journalisme narratif ${ }^{37}$, ou plus largement dans cette affection marquée du $x^{2}{ }^{e}$ siècle pour le document. Comme le remarquent Philippe Daros, Alexandre Gefen et Alexandre Prstojevic dans leur appel à communication pour un colloque consacré aux territoires de la non-fiction,

[s]i les siècles qui le précédaient avaient vu le couronnement du roman, la littérature $\mathrm{du} \mathrm{xxI}$ siècle débute avec le triomphe du document: écritures de voyage, d'investigation, enquêtes judiciaires ou ethnologiques, autobiographies, factographies, factions, rapports et enregistrements littéraires, et autres formes de récits refusant de se dire romans occupent nos librairies ${ }^{38}$.

Ce à quoi il faudrait ajouter nos journaux et nos réseaux. Cette non-fiction transmédia élaborée par Beaudoux contribue à son tour à brouiller genres du discours et disciplines. L'histoire même du journalisme littéraire procède de ce brouillage, dont Marc Lits retrace l'itinéraire « depuis les articles fondateurs de Tom Wolfe et le "roman vrai" de Truman Capote, dans Cold Blood (1966), en passant par le Colombien Gabriel Garcia Marquez, le Polonais Ryszard Kapuscinski ou le Français Jonathan Littell ${ }^{39}$ ». Le Madeleine Project possède bien des caractéristiques attribuées au journalisme littéraire tel que le décrit Norman $\operatorname{Sims}^{40}$ : immersion du reporter, accès et relation au sujet, attention marquée aux vies ordinaires, précision factuelle et en même temps importance de la voix, du style, construction complexe du texte et ultimement 
attention portée à la valeur symbolique de l'histoire. Alain Lallemand résume le journalisme narratif par ces quelques mots: «action, proximité, implication et pourtant distance critique; fiabilité, voix et structure signifiante ${ }^{41}$ ", Lallemand entendant par implication l'importance pour le journaliste de ne pas être seulement sur le terrain, mais de prendre part à l'action, de la vivre - ce que fait pleinement Beaudoux, notamment à travers la performativité de son récit. Lallemand désigne également par structure signifiante l'idée que le récit permet de dégager « une leçon de $v^{42}{ }^{42} »$, qu'il doit posséder une dimension sociale, entrer en résonnance avec la sociétée ${ }^{43}$. C'est ici que petite et grande histoire renouent, que documentation et narration littéraire s'entremêlent.

Le Madeleine Project, le journalisme narratif - tel que le définit Lallemand du moins apparaissent alors éminemment ricœuriens ; en ce sens qu'ils articulent, à l'image de ce que propose le philosophe dans Temps et récit, "l'expérience viscérale, tragique, de notre temporalité ${ }^{44}$ » et l'activité narrative dont l'enjeu est fondamentalement herméneutique. Le temps n'est saisissable que par les récits, les histoires et les mythes que nous racontons :

Le temps devient temps humain dans la mesure où il est articulé de manière narrative; en retour le récit est significatif dans la mesure où il dessine les traits de l'expérience temporelle ${ }^{45}$.

Nous lisons ou racontons des histoires, fictives ou non, comme celles de Madeleine, parce qu'elles donnent un sens à l'intrigue de nos vies. "C'est que notre vie est une histoire qui non seulement a besoin, mais qui mérite d'être racontée ${ }^{46}$ ", déclare Ricœur dans Temps et récit 3. Celle de Madeleine, qui n'a ni sépulture, ni enfant pour garder sa mémoire, méritait d'être racontée - «Ici écriture vaut sépulture », comme le déclare Jean Grondin ${ }^{47}$ à partir de l'analyse de Ricœur. Beaudoux s'impose alors en rempart contre "l'oubli d'effacement ${ }^{48}$ ", et ce quoi qu'en disent ses détracteurs sur le Net trouvant que la vie privée de Madeleine avait été bafouée. À l'opposé de cette «ligne brisée ${ }^{49}$ " qu'est "l'oubli d'effacement" peut se trouver une mémoire heureuse, ce bonheur qui nous vient quand une bribe du passé se trouve arrachée à l'oubli ${ }^{50}$. Voilà très exactement ce sur quoi repose le Madeleine Project. L'histoire de cette institutrice éclaire notre compréhension de nous-même, de notre identité, elle donne corps à ce que Ricœur nomme notre identité narrative. «[...] [R]épondre à la question "qui ?" c'est raconter l'histoire d'une vie ${ }^{51}$ ", écrit-il, et c'est ce qu'aura réalisé Beaudoux, un tweet après l'autre.

\section{BIBLIOGRAPHIE}

BACHIMONT Bruno, « Préface », in Matteo TRELEANI, Mémoires audiovisuelles, Montréal, Les Presses de l'Université de Montréal, 2014, p. 9-13. [En ligne] http://books.openedition.org/pum/2109 [consulté le 12 janvier 2018].

BARONI Raphaël, La Tension narrative. Suspense, curiosité et surprise, Paris, Seuil, 2007. 
BARTHES Roland, La Chambre claire, Paris, Gallimard, 1980.

BAUDRILLARD Jean, La Guerre du Golfe n'a pas eu lieu, Paris, Galilée, 1991.

BEAudoux Clara, Madeleine Project, Paris, Éditions du sous-sol, 2016.

BEAUdoux Clara et HiRsCHAUER Emmanuelle, « Grâce au Madeleine Project je me suis mise à dire je (entretien) », bibliobs, 28 mai 2016. [En ligne] https://bibliobs.nouvelobs.com/web-side-stories/ 20160527.OBS1387/grace-au-madeleine-project-je-me-suis-mise-a-dire-je.html [consulté le 18 mai 2017].

CARR Cynthia, On Edge. Performance at the End of the 20th Century [1993], Middletown, Wesleyan University Press, 2008.

CARRUTHERS Mary, Machina memorialis. Méditation, rhétorique et fabrication des images au Moyen Âge [1998], Paris, Gallimard, 2002.

DARos Philippe, Gefen Alexandre et PRSTOJEVIC Alexandre, " Territoires de la non-fiction », Fabula, 15 juin 2017. [En ligne] https://www.fabula.org/actualites/territoires-de-la-nonfiction_79215.php [consulté le 12 janvier 2018].

DUBIED Annick, « Le récit médiatique. Un objet complexe en quête de définition », Communication, vol. 19, 1999.

GOLDBERG Roselee, Performances. L'art en action, Londres, Thames \& Hudson, 1999.

GOLDBERG Roselee, La Performance, du futurisme à nos jours [1979], Londres, Thames \& Hudson, 2001.

GERVAIS Bertrand, La Ligne brisée. Labyrinthe, oubli et violence, Montréal, Le Quartanier, 2008.

GRONDIN Jean, Paul Ricour [2013], Paris, Presses Universitaires de France, 2016.

GUILET Anaïs, Pour une littérature cyborg : l'hybridation médiatique du texte littéraire, thèse de doctorat, Poitiers, Université de Poitiers/Université du Québec à Montréal, 28 mars 2013.

KRÄMER Sybille, «Qu'est-ce donc qu'une trace, et quelle est sa fonction épistémologique ? État des lieux », Trivium, $\mathrm{n}^{\circ}$ 10, 2012.

KüMMEL Christoph, « Wie weit trägt ein Indizienbeweis ? Zur archäologischen Überführung von Grabräubern », in Veit Ulrich (dir.), Spuren und Botschaften. Interpretationen materieller Kultur, Münster, Waxmann, 2003, p. 135-156.

LABEL-Rojoux Arnaud, Acte pour l'art [1989], Paris, Al Dante, 2007.

LALLEMAND Alain, Journalisme narratif en pratique, Bruxelles, De Boeck, 2011.

LITS Marc, Du récit au récit médiatique, Bruxelles, De Boeck, 2008.

LITS Marc, «Quel futur pour le récit médiatique ?», Questions de communication, n² 21, 2012. [En ligne] https://journals.openedition.org/questionsdecommunication/6562 [consulté le 30 septembre 2016].

PARRET Herman, « Vestige, archive et trace. Présences du temps passé », Protée, n 322, 2004, p. 37-46.

Proust Marcel, Du côté de chez Swann [1913], Paris, Flammarion, 1987.

RICceUR Paul, Temps et récit 1, Paris, Seuil, 1983.

RICEURR Paul, Temps et récit 2, Paris, Seuil, 1984.

RICEUUR Paul, Temps et récit 3, Paris, Seuil, 1991. 
RICœUR Paul, La Mémoire, l'histoire l'oubli, Paris, Seuil, 2000.

SALMON Christian, Storytelling. La machine à fabriquer des histoires et à formater les esprits, Paris, La Découverte, 2007.

SIMS Norman, True Stories. A Century of Literary Journalism, Evanston, Northwestern University Press, 2008.

VIRILIo Paul, L'Écran du désert. Chroniques de guerre, Paris, Galilée, 1991.

VIRILIO Paul, L'Administration de la peur, Paris, Textuel, 2010.

\section{NOTES}

1. Herman Parret, «Vestige, archive et trace. Présences du temps passé », Protée, n 322, 2004, p. 43.

2. Clara Beaudoux, Madeleine Project. [En ligne] https://twitter.com/clarabdx [consulté le 12 janvier 2018].

3. Clara Beaudoux, Madeleine Project, Paris, Éditions du sous-sol, 2016, p. 7.

4. Clara Beaudoux, Madeleine Project. [En ligne] http://madeleineproject.fr [consulté le 12 janvier 2018].

5. Clara Beaudoux, Madeleine Project. [En ligne] https://storify.com/clarabdx/madeleineproject [consulté le 12 janvier 2018].

6. Herman Parret, «Vestige, archive et trace. Présences du temps passé », Protée, n 322, 2004, p. 43.

7. Herman Parret, «Vestige, archive et trace. Présences du temps passé », Protée, n 322, 2004, p. 43.

8. Roland Barthes, La Chambre claire, Paris, Gallimard, 1980.

9. Herman Parret, «Vestige, archive et trace. Présences du temps passé », Protée, n 322, 2004, p. 43.

10. Sybille Krämer, «Qu'est-ce donc qu'une trace, et quelle est sa fonction épistémologique ? État des lieux », Trivium, $\mathrm{n}^{\circ}$ 10, 2012.

11. Jean Baudrillard, La Guerre du Golfe n'a pas eu lieu, Paris, Galilée, 1991.

12. Paul Virilio, L'Écran du désert. Chroniques de guerre, Paris, Galilée, 1991.

13. Paul Virilio, L'Administration de la peur, Paris, Textuel, 2010, p. 8.

14. Cf. Roselee Goldberg, Performances. L'art en action, Londres, Thames \& Hudson, 1999 ; Roselee Goldberg, La Performance, du futurisme à nos jours [1979], Londres, Thames \& Hudson, 2001 ; Cynthia Carr, On Edge. Performance at the End of the 20th Century [1993], Middletown, Wesleyan University Press, 2008 ; Arnaud Label-Rojoux (1989), Acte pour l'art, Paris, Al Dante, 2007.

15. Clara Beaudoux, Madeleine Project, Paris, Éditions du sous-sol, 2016, p. 9.

16. Christian Salmon, Storytelling. La machine à fabriquer des histoires et à formater les esprits, Paris, La Découverte, 2007.

17. Anaïs Guilet, Pour une littérature cyborg: l'hybridation médiatique du texte littéraire, thèse de doctorat, Poitiers, Université de Poitiers/Université du Québec à Montréal, 28 mars 2013.

18. Clara Beaudoux, « Préface éditoriale », Madeleine Project, Paris, Éditions du sous-sol, 2016, p. 7.

19. Christoph Kümmel, «Wie weit trägt ein Indizienbeweis? Zur archäologischen Überführung von Grabräubern », in Ulrich Veit (dir.), Spuren und Botschaften. Interpretationen materieller Kultur, Münster, Waxmann, 2003, p. 143.

20. Bruno Bachimont, "Préface », in Matteo Treleani (dir.), Mémoires audiovisuelles, Montréal, Les Presses de l'Université de Montréal, 2014, p. 9-13. [En ligne] http://books.openedition.org/ pum/2109 [consulté le 12 janvier 2018]. 
21. Page de présentation du Madeleine Project sur le site des Éditions du sous-sol. [En ligne] http:// www.editions-du-sous-sol.com/publication/madeleine-Project/ [consulté le 12 janvier 2018].

22. Marcel Proust, Du côté de chez Swann [1913], Paris, Flammarion, 1987, p. 140-145.

23. Cf. Paul Ricoeur, Temps et récit 2, Paris, Seuil, 1984.

24. Voir la définition du récit par Annick Dubied. Résumé en dix critères : début, milieu et fin ; étendue appropriée; principe de causalité narrative; principe de nécessité narrative; thème ; implication d'intérêts humains ; conclusion imprévisible et congruante ; conclusion qui permet la rétrospection; inversion de l'effet des actions; actualisation (Annick Dubied, «Le récit médiatique. Un objet complexe en quête de définition ", Communication, vol. 19, 1999).

25. Nous pensons ici notamment à L'Hôtel (1981) ou Le Carnet d'adresse (1983).

26. Clara Beaudoux, « Préface éditoriale », Madeleine Project, Paris, Éditions du sous-sol, 2016, p. 7.

27. Raphaël Baroni, La Tension narrative. Suspense, curiosité et surprise, Paris, Seuil, 2007.

28. Clara Beaudoux, Madeleine Project, Paris, Éditions du sous-sol, 2016, p. 118.

29. Clara Beaudoux, Madeleine Project, Paris, Éditions du sous-sol, 2016, p .47.

30. Clara Beaudoux, Madeleine Project, Paris, Éditions du sous-sol, 2016, p. 63.

31. Clara Beaudoux, Madeleine Project, Paris, Éditions du sous-sol, 2016, p. 30.

32. Clara Beaudoux et Emmanuelle Hirschauer, «Grâce au Madeleine Project je me suis mise à dire je (entretien) », bibliobs, 28 mai 2016. [En ligne] https://bibliobs.nouvelobs.com/web-sidestories/20160527.OBS1387/grace-au-madeleine-project-je-me-suis-mise-a-dire-je.html [consulté le 18 mai 2017].

33. Clara Beaudoux, Madeleine Project, Paris, Éditions du sous-sol, 2016, p. 5.

34. Mary Carruthers, Machina memorialis. Méditation, rhétorique et fabrication des images au Moyen Âge [1998], Paris, Gallimard, 2002, p. 17.

35. Herman Parret, « Vestige, archive et trace. Présences du temps passé », Protée, n 322, 2004, p. 40.

36. Clara Beaudoux, Madeleine Project, Paris, Éditions du sous-sol, 2016.

37. Journalisme narratif qui lui-même s'inscrit dans la lignée du grand reportage à la française tel que l'a inauguré Albert Londres.

38. Philippe Daros, Alexandre Gefen et Alexandre Prstojevic, "Territoires de la non-fiction ", Fabula, 15 juin 2017. [En ligne] https://www.fabula.org/actualites/territoires-de-la-nonfiction_79215.php [consulté le 12 janvier 2018].

39. Marc Lits, "Quel futur pour le récit médiatique?", Questions de communication, $\mathrm{n}^{\circ} 21,2012$. [En ligne] https://journals.openedition.org/questionsdecommunication/6562 [consulté le 30 septembre 2016].

40. Norman Sims, True Stories. A Century of Literary Journalism, Evanston, Northwestern University Press, 2008.

41. Alain Lallemand, Journalisme narratif en pratique, Bruxelles, De Boeck, 2011, p. 23.

42. Alain Lallemand, Journalisme narratif en pratique, Bruxelles, De Boeck, 2011, p. 23.

43. Cf. Marc Lits, Du récit au récit médiatique, Bruxelles, De Boeck, 2008.

44. Jean Grondin, Paul Ricœur [2013], Paris, Presses Universitaires de France, 2016.

45. Paul Ricœur, Temps et récit 1, Paris, Seuil, 1983, p. 17.

46. Paul Ricœur, Temps et récit 3, Paris, Seuil, 1991, p. 115.

47. Jean Grondin, Paul Ricœur [2013], Paris, Presses Universitaires de France, 2016.

48. Paul Ricœur, La Mémoire, l'histoire l'oubli, Paris, Seuil, 2000.

49. Bertrand Gervais, La Ligne brisée. Labyrinthe, oubli et violence, Montréal, Le Quartanier, 2008.

50. Paul Ricœur, Temps et récit 1, Paris, Seuil, 1983, p. 542.

51. Paul Ricœur, Temps et récit 3, Paris, Seuil, 1991, p. 355. 


\section{RÉSUMÉS}

Clara Beaudoux trouve dans la cave de son nouvel appartement les affaires de Madeleine, l'ancienne locataire des lieux. Pendant une semaine, sur Twitter, elle mènera l'enquête avec tendresse et pudeur : quelques mots, des images, le portrait de Madeleine en même temps que celui d'une époque révolue, se dessinent. À travers sa performance sur les réseaux et dans une démarche proche du journalisme narratif, les archives de Madeleine se constituent: un «[...] archivage, comme une mise à nu ${ }^{1}{ }$ qui permet d'interroger la mémoire mais surtout la trace, qui se fait ici poétique. Le Madeleine Project en cela est résolument proustien, il procède de cette réminiscence aussi nostalgique qu'actuelle qui, dans le cadre du projet, se développe d'abord via les réseaux sociaux pour aboutir à un livre publié en 2016: Beaudoux, à l'image de Marcel, devenant ainsi écrivaine.

\section{INDEX}

Mots-clés : archive, journalisme narratif, mémoire, performance, récit, réseaux sociaux

\section{AUTEUR}

\section{ANAÏS GUILET}

Anaïs Guilet est maîtresse de conférences en littératures comparées et en sciences de l'information et de la communication à l'Université Savoie Mont Blanc. Elle est rattachée au laboratoire de recherche LLSETI où elle codirige l'équipe 3 de l'axe 2 consacré au « Corps en question ». Elle est membre associée du laboratoire FIGURA, à l'UQAM. Spécialisée dans les humanités numériques, ses recherches portent sur les esthétiques et poétiques numériques et transmédiatiques, sur la place du livre dans la culture contemporaine ainsi que sur les représentations du corps en contexte numérique. Site web : www.cyborglitteraire.com. 